\title{
On the Efficiency and Sensitivity of a Pyramidal Classification Algorithm
}

\author{
Angel J. Gil ${ }^{1} \quad$ Carles Capdevila $^{2} \quad$ Antoni Arcas $^{3}$
}

${ }^{1}$ Departament d'Economia i Empresa. Universitat Pompeu Fabra, Barcelona. gil_angeleempr.upf.es

${ }^{2}$ Departament de Matemàtica, Universitat de Lleida. ccm@matematica.udl.es

${ }^{3}$ Departament d'Estadística, Universitat de Barcelona. 


\begin{abstract}
In this paper we propose a Pyramidal Classification Algorithm, which together with an appropriate aggregation index produces an indexed pseudo-hierarchy (in the strict sense) without inversions nor crossings. The computer implementation of the algorithm makes it possible to carry out some simulation tests by Monte Carlo methods in order to study the efficiency and sensitivity of the pyramidal methods of the Maximum, Minimum and UPGMA. The results shown in this paper may help to choose between the three classification methods proposed, in order to obtain the classification that best fits the original structure of the population, provided we have an a priori information concerning this structure.
\end{abstract}

Keywords: Pyramidal classification methods, aggregation index, pseudo-hierarchy, Robinsonian dissimilarity, Monte Carlo evaluation, overlapping clusters

Journal of Economic Literature Classification: C15, C88 


\section{Introduction}

Ultrametric trees are the most studied representations using discrete models. The aim of this class of models is to produce a family of partitions that can be interpreted as a set of "natural" classifications of a given population.

Pyramidal trees, introduced by E. Diday, are a logical generalization of ultrametric trees. In the pyramidal model, each classification is given by a set of overlapping clusters, instead of a partition. The pyramidal representation being less restrictive, it is closer to the initial structure of the population than in the hierarchical case. Bertrand and Diday [BD85], Diday [Did86b, Did86a], Durand [Dur86, Dur88], Durand and Fichet [DF88] have studied some interesting topics about this pyramidal structures, also called pseudo-hierarchical structures.

The aim of the pyramidal representation methods is to detect the presence of a pseudo-hierarchical structure on a given population $\Omega$, starting from a dissimilarity matrix on this population. The process that has this aim consists of transforming the initial dissimilarity into a Robinsonian dissimilarity by means of a pyramidal clustering procedure; this Robinsonian dissimilarity obtained is equivalent to an indexed pseudo-hierarchy.

Diday, inspired by the agglomerative hierarchical classification algorithms, proposes (see [Did86b]) an agglomerative pyramidal classification algorithm which is adapted to the peculiar characteristics of the pyramidal structures; among these we can point out the fact that each group can have up to two predecessors, as well as the existence of a total order on the population to be classified which is compatible with the pseudo-hierarchy. In general, this algorithm produces indexed pseudo-hierarchies in a wide sense, which may present inversion problems. In our work we propose a modification of Diday's algorithm that makes possible the construction of an indexed pseudo-hierarchy in the strict sense and without inversion problems. So the algorithm described in this paper produces simpler pseudo-hierarchies than Diday's one. This new algorithm, which we call PIRAM, was introduced in [CGA95]. We think that the new features of our algorithm will facilitate the application of the pyramidal classification methods in real problems.

In applied problems it is necessary to measure the fitting between the pseudohierarchical structure obtained by using some algorithm and the initial structure of the population. This fitting can be measured by comparing the dissimilarity $\delta$, associated with the initial structure, and the dissimilarity $d$, associated with the structure produced by the algorithm. ${ }^{1}$ This comparison can be made by using a number of different approaches (see [Gor96]). In this paper we will concentrate on the analysis of the behaviour of the following coefficients:

- The $\gamma$-Goodman-Kruskal coefficient defined in [GK54] as

$$
\gamma=\frac{\left(S_{+}-S_{-}\right)}{\left(S_{+}+S_{-}\right)},
$$

where $S_{+}, S_{-}$are the number of pairs of couples $\{i, j\},\{r, s\}$ with $\left(d_{i j}-\right.$ $\left.d_{r s}\right)\left(\delta_{i j}-\delta_{r s}\right)>0$, or $<0$ respectively (concordant resp. discordant pairs); This coefficient is interpreted in [Bak74] in the following way:

\footnotetext{
${ }^{1}$ The distance $d$ between two individuals in the pseudo-hierarchy is the level at which both individuals join the same cluster; this kind of distances are sometimes called threshold distances.
} 
$\gamma=$ probability of consistent ranking- probability of inconsistent

ranking.

A modification of this coefficient was used in [Hub74] to implement a stopping rule (see [Gor96]).

- The cophenetic correlation coefficient defined in [SR62] as

$$
\rho=\frac{\operatorname{Cov}(d, \delta)}{\sigma(d) \sigma(\delta)}
$$

Furthermore, the computer implementation of the algorithm PIRAM (see [GCA94a], [GCA94b] and [GC96]), called PIRAM1.0, has allowed us to compare the pyramidal and the "classical" hierarchical classification methods (see [CGA95]). We have also compared three different pyramidal methods-Maximum, Minimum, and UPGMA- associated with three different ways of defining the distance between clusters (aggregation index). In this paper we present the simulation tests by Monte Carlo methods produced in order to study the efficiency and sensitivity of these pyramidal methods in an empirical way and in terms of the expected mean value of the $\gamma$ and $\rho$ coefficients (see [CA95a] and [CGA96]).

\section{Pyramidal models}

In this section we will introduce some concepts related to pyramidal models, such as Robinsonian dissimilarity and pseudo-hierarchy.

Given a dissimilarity $d$, defined on a finite set $\Omega$, we will call it a Robinsonian dissimilarity if it is a symmetric dissimilarity such that there exists a total order $\leq_{\Omega}$ on $\Omega$, such that

$$
\max \left\{d\left(\omega_{i}, \omega_{j}\right), d\left(\omega_{j}, \omega_{k}\right)\right\} \leq d\left(\omega_{i}, \omega_{k}\right)
$$

for any $\omega_{i}, \omega_{j}, \omega_{k} \in \Omega$ such that $\omega_{i} \leq_{\Omega} \omega_{j} \leq_{\Omega} \omega_{k}$. From this definition it is easy to see that any ultrametric dissimilarity is also a Robinsonian dissimilarity (see [Did86b]).

Given a finite set $\Omega$ and $\mathbf{P} \in \operatorname{Pow}(\Omega)$, we will say that $\mathbf{P}$ is a pseudohierarchy, if it satisfies the following properties:

P.1 $\Omega \in \mathbf{P}$.

P.2 for all $\omega \in \Omega,\{\omega\} \in \mathbf{P}$.

P.3 for all $h, h^{\prime} \in \mathbf{P}, h \cap h^{\prime}=\emptyset$ or $h \cap h^{\prime} \in \mathbf{P}$.

P.4 There exists a pre-order $\leq_{\mathbf{P}}$ on $\Omega$ such that every $h \in \mathbf{P}$ is an interval with respect to $\leq_{\mathbf{P}}$. We say that $\leq_{\mathbf{P}}$ is an order compatible with $\mathbf{P}$.

Note that $\mathbf{P} \in \operatorname{Pow}(\Omega)$ is a hierarchy if it satisfies [P.1], [P.2] and the following properties:

P.5 for all $h, h^{\prime} \in \mathbf{P}, h \cap h^{\prime} \in\left\{\emptyset, h, h^{\prime}\right\}$.

P.6 For all $h \in \mathbf{P}, \cup\left\{h^{\prime} \in \mathbf{P}: h^{\prime} \subseteq h\right\} \in\{h, \emptyset\}$. 
Since, given a hierarchy, there is always an order on the population compatible with it, we can conclude that a hierarchy is a pseudo-hierarchy (see [Did86b]). See also [GS94] for a deeper analysis of the relations between ultrametric dissimilarities, Robinsonian dissimilarities, hierarchies and pyramids.

Given two groups of a pseudo-hierarchy, $h$ and $h^{\prime}$, we say that $h^{\prime}$ is a predecessor of $h$ when $h \subset h^{\prime}$ and there is no other group $p$ in the pseudo-hierarchy such that $h \subset p \subset h^{\prime}$. In can be proved that any group in a pseudo-hierarchy can have up to two predecessors, while in a hierarchy every group has only one predecessor (see [Cap93]).

Given $\mathbf{P}$ as a pseudo-hierarchy on $\Omega$, a function $i: \mathbf{P} \mapsto R^{+}$, such that for all $\omega \in \Omega, i(\{\omega\})=0$ and for all $h, h^{\prime} \in \mathbf{P}$, if $h \subseteq h^{\prime}$ then $i(h) \leq i\left(h^{\prime}\right)$, is called an index associated with $\mathbf{P}$, and the pair $(\mathbf{P}, i)$ is called an indexed pseudohierarchy. If, in addition, the function $i$ verifies that for all $h, h^{\prime} \in \mathbf{P}$, if $h \subset h^{\prime}$ then $i(h)<i\left(h^{\prime}\right)$, we will say that $(\mathbf{P}, i)$ is an indexed pseudo-hierarchy in the strict sense. The elements in a pseudo-hierarchy will be called groups of the pseudo-hierarchy, and if a pseudo-hierarchy is indexed, the value of the index over each group will be called index of the group.

If $(\mathbf{P}, i)$ is an indexed pseudo-hierarchy on $\Omega$, the dissimilarity associated with $(\mathbf{P}, i)$ is defined as the threshold distance, i.e.,

$$
d\left(\omega_{1}, \omega_{2}\right)=i(h)
$$

where $h$ is the smallest (with respect to the inclusion relation) $h \in \mathbf{P}$ such that $\omega_{1} \in h$ and $\omega_{2} \in h$ (see [Cap93]).

The relationship between Robinsonian dissimilarities and pseudo-hierarchies is studied, among others, in [Did86a] and in [CA95b] where the following results can be found:

Theorem 2.1 If $(\mathbf{P}, i)$ is an indexed pseudo-hierarchy on $\Omega$, then the dissimilarity associated with $(\mathbf{P}, i)$ is a Robinsonian dissimilarity.

Remark 2.1 Every Robinsonian dissimilarity determines an indexed pseudohierarchy, which is unique, so we can conclude that there exists a natural bijection between the set of indexed pseudo-hierarchies and the set of Robinsonian dissimilarities on a set $\Omega$.

The type of overlapping clustering associated with an indexed pseudo-hierarchy can be represented graphically in a pyramid-like shape by using a type of connex graphs called pyramidal trees (for this reason sometimes we write pyramid for pseudo-hierarchy). The visual representation of Indexed Hierarchies is provided by the well known dendrograms. Let us see some examples

Example 2.1 Let $\Omega=\left\{\omega_{1}, \omega_{2}, \omega_{3}, \omega_{4}, \omega_{5}\right\}$, with $\omega_{1} \leq \omega_{2} \leq \omega_{3} \leq \omega_{4} \leq \omega_{5}$. Let

$$
\begin{gathered}
\mathbf{P}=\left\{\left\{\omega_{1}\right\}, \ldots,\left\{\omega_{5}\right\},\left\{\omega_{2}, \omega_{3}\right\},\left\{\omega_{3}, \omega_{4}\right\},\left\{\omega_{1}, \omega_{2}, \omega_{3}\right\},\left\{\omega_{3}, \omega_{4}, \omega_{5}\right\},\right. \\
\\
\left.\left\{\omega_{2}, \omega_{3}, \omega_{4}\right\},\left\{\omega_{2}, \omega_{3}, \omega_{4}, \omega_{5}\right\},\left\{\omega_{1}, \omega_{2}, \omega_{3}, \omega_{4}\right\}, \Omega\right\}
\end{gathered}
$$

if we define

$$
\begin{array}{ll}
i\left(\left\{\omega_{j}\right\}\right)=0, \text { for } j \in\{1, \ldots, 5\} & i\left(\left\{\omega_{2}, \omega_{3}, \omega_{4}\right\}\right)=5 \\
i\left(\left\{\omega_{2}, \omega_{3}\right\}\right)=2 & i\left(\left\{\omega_{2}, \omega_{3}, \omega_{4}, \omega_{5}\right\}\right)=5 \\
i\left(\left\{\omega_{3}, \omega_{4}\right\}\right)=2.5 & i\left(\left\{\omega_{1}, \omega_{2}, \omega_{3}, \omega_{4}\right\}\right)=6 \\
i\left(\left\{\omega_{1}, \omega_{2}, \omega_{3}\right\}\right)=3 & i(\Omega)=7 \\
i\left(\left\{\omega_{3}, \omega_{4}, \omega_{5}\right\}\right)=4, &
\end{array}
$$


then $\mathbf{P}$ is an indexed pseudo-hierarchy.

If we start from the singletons, $\left\{\omega_{1}\right\}, \ldots,\left\{\omega_{5}\right\}$ and we determine their successive predecessors, we obtain the graphical representation of the pseudo-hierarchy displayed in Figure 1, and a representation of the indexed pseudo-hierarchy $(\mathbf{P}, i)$ displayed in a pyramidal form in Figure 2.

Figure 1: A pseudo-hierarchy

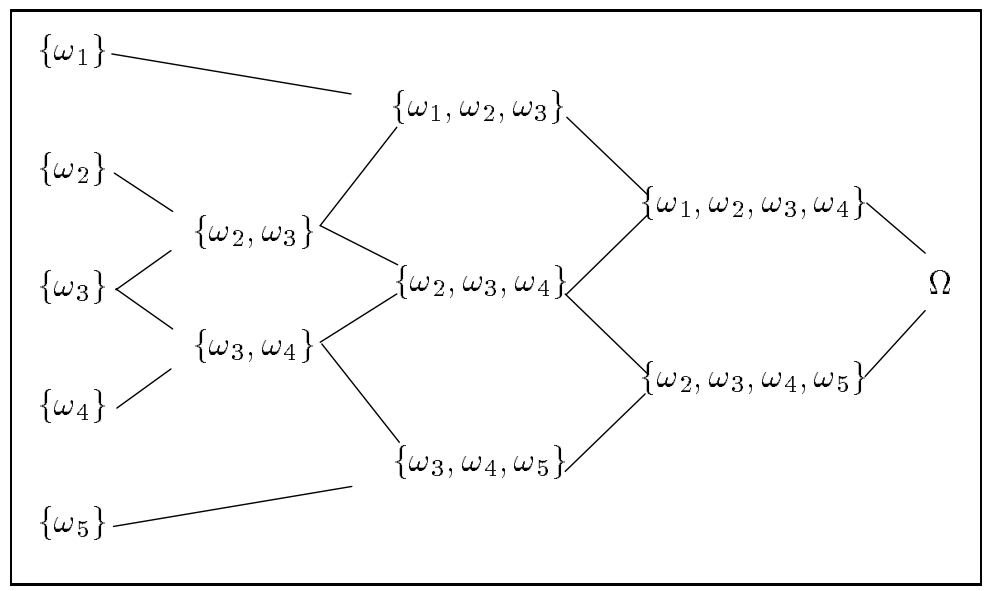

Example 2.2 Note that, if we proceed as in the hierarchical case, the pyramidal classification obtained from the pyramidal tree shown in Figure 2 at level 4, and which correspond to the vertical lines crossed by the horizontal line drawn at that level, are the following: $\left\{\omega_{1}, \omega_{2}, \omega_{3}\right\},\left\{\omega_{2}, \omega_{3}\right\},\left\{\omega_{3}, \omega_{4}\right\},\left\{\omega_{3}, \omega_{4}, \omega_{5}\right\}$, which, obviously, is a set of overlapping clusters.

Figure 2: Pyramidal graph of the pseudo-hierarchy of Example 2.1

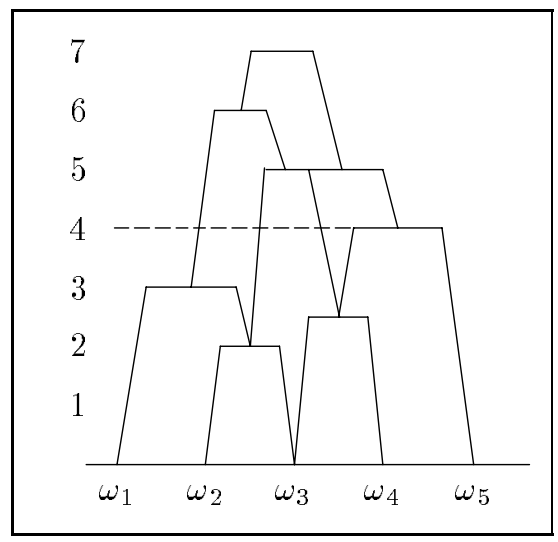


We introduce now two concepts: a function $f: \mathbf{P} \mapsto R^{+}$on a pseudohierarchy $\mathbf{P}$ presents an inversion if there exist $h, h^{\prime} \in \mathbf{P}$ such that $h \subset h^{\prime}$ and $f(h)>f\left(h^{\prime}\right)$. An order $\leq$ on $\Omega$ originates a crossing over a set $\mathbf{P} \in$ $\operatorname{Pow}(\Omega)$ either if there exists a group $h \in \mathbf{P}$ which is not an interval with respect to this order or there exist groups $h, h^{\prime} \in \mathbf{P}$ such that $h \cap h^{\prime} \neq \emptyset$ and $h \cap h^{\prime} \notin \mathbf{P}$. Examples of pyramidal-like shapes that present inversion or crossings are displayed in Figure 3. Note that due to its definition, the index of a pseudo-hierarchy cannot present inversions and an order compatible with a pseudo-hierarchy cannot present crossings. Nevertheless, these concepts which, in fact, are implicit in the definition of a pseudo-hierarchy, should be considered in the process of generating pseudo-hierarchies from dissimilarity matrices.

Figure 3: (A) Inversion: $h \subset h^{\prime}$ and $i\left(h^{\prime}\right)<i(h)$; (B) Crossing: $h \cap h^{\prime}=$ $\left(\omega_{2}, \omega_{3}, \omega_{4}\right) \neq \emptyset$ but $h \cap h^{\prime} \notin \mathbf{P} ;(\mathrm{C}) h=\left(\omega_{2}, \omega_{4}\right) \in \mathbf{P}$ is not an interval w.r.t. the order associated with the pseudo-hierarchy.

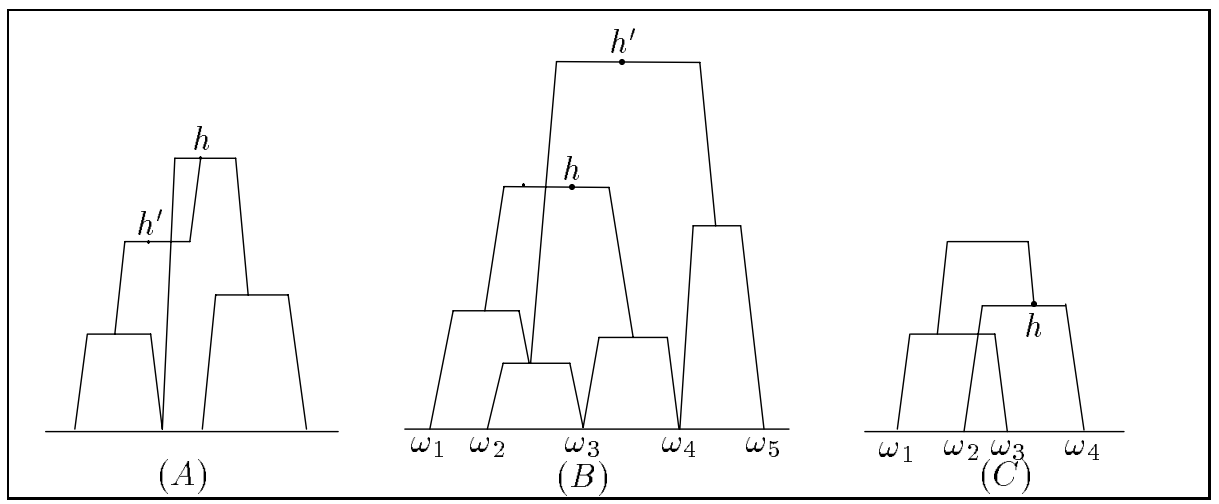

\section{PIRAM, a Pyramidal Classification Algorithm}

Let $\Omega=\left\{\omega_{1}, \ldots, \omega_{n}\right\}$ be a finite set and let $\delta_{0}$ be a dissimilarity defined on the individuals of $\Omega$. Like in the agglomerative hierarchical case, the algorithm we present builds a sequence of levels in such a way that the $(i+1)-t h$ level is obtained by joining two groups among the groups obtained at the $i-t h$ level. In order to compute the distance between groups, an aggregation index should be chosen. Since the two groups joined may overlap, additional conditions should be stated to build up an order on $\Omega$ which is intended to be compatible with the final pseudo-hierarchy. The process will continue until all the individuals of the population belong to a single group.

So, after having fixed the function $f$ in (1), the algorithm of pyramidal classification PIRAM described hereinafter produces

- a sequence $R_{0}, \ldots, R_{m}$, each $R_{i}$ being a set of overlapping classes of elements of $\Omega$, in such a way that $\mathbf{P}=\cup R_{i}$ is a pseudo-hierarchy;

- a sequence of dissimilarities $\delta_{0}, \delta_{1}, \ldots, \delta_{m}$, each $\delta_{i}$ defined on the set $R_{i}$; 
- a sequence $\leq_{0}, \leq_{1}, \ldots \leq_{m}=\leq_{\mathbf{P}}$ of partial linear orders on the set $\Omega$ such that $\leq_{\mathbf{P}}$ is a total order on $\bar{\Omega}$, every $h \in R_{i}$ is an interval with respect to $\leq_{i}$, and $\leq_{i+1}$ extends $\leq_{i}$, i.e., if $\omega_{j} \leq_{i} \omega_{k}$ then $\omega_{j} \leq_{i+1} \omega_{k}$;

- an increasing sequence of real numbers $r_{0}, \ldots, r_{m}$ which will correspond with the range of the index associated with the pseudo-hierarchy $\mathbf{P}$.

Note that the existence of an order compatible with the pseudo-hierarchy $\mathbf{P}$ makes it possible to interpret the groups of $\mathbf{P}$ as $\leq \mathbf{P}^{-i n t e r v a l s}$ and, in fact, each of its groups can be displayed as a sequence of distinct elements of $\Omega$. So, when merging two groups, the set-theoretical operation "join" can be seen as a certain kind of concatenation operation between sequences. So we will consider the following operation:

If $h_{i}=\left(\alpha_{1}, \ldots, \alpha_{n}\right) \in \Omega^{n}$ and $h_{j}=\left(\beta_{1}, \ldots, \beta_{l}\right) \in \Omega^{l}$ then

$$
h_{i}+h_{j}= \begin{cases}\left(\alpha_{1}, \ldots, \alpha_{n}, \beta_{1}, \ldots, \beta_{l}\right) & \text { if } h_{i} \cap h_{j}=\emptyset \\ \left(\alpha_{1}, \ldots, \alpha_{n}, \beta_{k}, \ldots, \beta_{l}\right) & \text { if } h_{j}=\left(\alpha_{s}, \ldots, \alpha_{n}, \beta_{k}, \ldots, \beta_{l}\right) \text { and } \\ & \left(\alpha_{1}, \ldots, \alpha_{s-1}\right) \cap h j=\emptyset \\ \text { not defined } & \text { in any other case }\end{cases}
$$

We describe now the steps of the algorithm:

A1. The procedure starts with the set $R_{0}=\left\{\left\{\omega_{1}\right\}, \ldots,\left\{\omega_{n}\right\}\right\}$, and the dissimilarity $\delta_{0}$. Let $r_{0}=0$ and let $\leq_{0}$ be the partial order defined by $\omega_{i} \leq_{0} \omega_{j}$ iff $i=j$. For any $\omega \in \Omega$ we define $i(\{\omega\})=0$.

Given $R_{k-1}, \delta_{k-1}, \leq_{k-1}$ and $r_{k-1}$, we consider $i_{k}=r_{k-1}$ and

A2. We choose a pair $\left(h_{i}, h_{j}\right) \in R_{k-1}^{2}$ such that $\delta_{k-1}\left(h_{i}, h_{j}\right)=i_{k}$ and it satisfies the following conditions

A2.1 $h_{i}+h_{j}$ is defined.

A2.2 there is no other pair $\left(h_{i}^{*}, h_{j}^{*}\right)$ such that $h_{i} \subset h_{i}^{*}, h_{j} \subset h_{j}^{*}$ and $\delta_{k-1}\left(h_{i}^{*}, h_{j}^{*}\right)=i_{k}$.

A2.3 there is a partial linear order on $\Omega, \leq_{k}$, such that all the elements in $R_{k-1} \cup\left\{h_{i}+h_{j}\right\}$ are intervals with respect to $\leq_{k}$ and such that $\leq_{k}$ extends $\leq_{k-1}$.

A2' If no pair satisfies A2.1, A2.2 and A2.3 then we set $a=i_{k}$ and we consider a new value of $i_{k}:=\min \left\{\delta_{k-1}\left(h_{r}, h_{s}\right): \delta_{k-1}\left(h_{r}, h_{s}\right)>a, \quad h_{r}, h_{s} \in R_{k-1}\right\}$ and we go back to step A2.

A3. Assume that the pair $\left(h_{i}, h_{j}\right)$ chosen in step A2 satisfies conditions A2.1, A2.2, and A2.3. We say that $h_{i}$ and $h_{j}$ have been joined. We define $h_{n+k}=h_{i}+h_{j}, i\left(h_{n+k}\right)=i\left(h_{i}+h_{j}\right)=\delta_{k-1}\left(h_{i}, h_{j}\right)$ and $r_{k}=\delta_{k-1}\left(h_{i}, h_{j}\right)$.

To build the set $R_{k}$ we consider the set $R_{k}^{*}=R_{k-1} \cup\left\{h_{i}+h_{j}\right\}$ and

A3.1 we delete $h_{i}$ from $R_{k}^{*}$, if $h_{i}$ has been joined twice;

A3.2 we delete $h_{j}$ from $R_{k}^{*}$, if $h_{j}$ has been joined twice;

A3.3 we delete from $R_{k}^{*}$ all those elements $h_{r}$ in $R_{k-1}$ such that $i\left(h_{r}\right)=$ $i\left(h_{i}+h_{j}\right)$. 
A3.4 we delete from $R_{k}^{*}$ all those elements $h_{r}$ in $R_{k-1}$ such that $h_{r} \subset h_{i}+h_{j}$ and $h_{r}$ contains none of the extrema of $h_{i}+h_{j}$ (see Figure 4).

Finally, a new dissimilarity $\delta_{k}$ is defined between the groups of the set $R_{k}$ in the following way:

$$
\begin{aligned}
& \delta_{k}\left(h, h^{\prime}\right)= \\
& \begin{cases}0 & \text { if } h \cap h^{\prime} \in\left\{h, h^{\prime}\right\} \\
\delta_{k-1}\left(h, h^{\prime}\right) & \text { if } h, h^{\prime} \in R_{k-1} \\
\max \left\{f\left(\delta_{k-1}\left(h, h_{i}\right), \delta_{k-1}\left(h, h_{j}\right), \ldots\right), i\left(h^{\prime}\right)\right\} & \text { if } h^{\prime}=h_{n+k}=h_{i}+h_{j}\end{cases}
\end{aligned}
$$

where $f$ is the function that determines the pyramidal method used, and which has to be the same within all the process. Usually (see [KR90]) we consider $f=\max$ (Maximum Method which yields the complete link pyramidal method) or $f=\min$ (Minimum Method which yields the single link pyramidal method) or $f=U P G M A$ (UPGMA method, see $[$ SM58]).

A4. Steps A.2 and A.3 are iterated until $h_{i}+h_{j}$ contains all the elements of $\Omega$.

Figure 4: Step A3.4. The extrema of $h_{s}$ are $\omega_{1}$ and $\omega_{4}$; the extrema of $h_{r}$ are $\omega_{2}$ and $\omega_{3} ; h_{j}=\left\{\omega_{4}\right\}$.

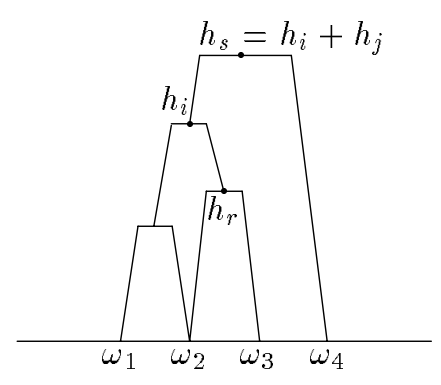

Note that by conditions A3.1 and A3.2 each group in the pseudo-hierarchy can only be joined twice. Thus each group can have at most two predecessors.

Conditions A2.2 and A3.3 are the basic points which differentiate our algorithm from Diday's one. Thanks to these conditions, the algorithm becomes faster and the pseudo-hierarchy produced is indexed in the strict sense as shown in [CGA95]. In addition, as we will see in Theorem 3.1, the pseudo-hierarchy built by the algorithm introduced does not present inversions nor crossings, and therefore the inversion problems of Diday's algorithm are solved.

Theorem 3.1 ([CGA95]) The algorithm PIRAM of pyramidal classification, together with the clustering index defined in (1), builds an indexed pseudohierarchy without crossings nor inversions.

Proof: It is straightforward to see that this pyramidal classification algorithm builds an indexed pseudo-hierarchy. The proof is similar to the proof that Diday's algorithm also builds an indexed pseudo-hierarchy (see [Did86b, Did86a]), and will be omitted here. 
Due to condition A2.3, the order $\leq_{\mathbf{P}}$ is a total order on $\Omega$ such that all the elements of the pseudo-hierarchy are intervals with respect to this order. Due to condition A3.4, the group $h_{r}$, which is deleted from $R_{k}^{*}$, will never be joined in a posterior step of the algorithm. This two facts make the pseudo-hierarchy crossings-free, that is, the resulting indexed pseudo-hierarchy will not present crossings (see Figure 4).

Concerning inversion, by $\mathbf{A} 2$ ', the definition of $\delta_{k}\left(h, h^{\prime}\right)$ in (1) ${ }^{2}$ and the use of the sequence $r_{0}, \ldots, r_{m}$, the pseudo-hierarchy will also be inversion-free.

From this algorithm we have developed a system for pyramidal classification (called PIRAM1.0) such that, starting from the number of individuals to classify and a matrix containing the dissimilarities between them, the system classifies individuals by the Minimum, Maximum and UPGMA methods, giving as an output an indexed pseudo-hierarchy in the strict sense, and the corresponding pyramidal tree. As fitting measurements between the pyramidal structure obtained and the initial structure of population, the correlation coefficient $\rho$ and the $\gamma$ coefficient of Goodman-Kruskal are calculated, both defined between the pyramidal dissimilarity obtained and the initial dissimilarity.

\section{Monte Carlo evaluation of the pyramidal clas- sification methods.}

Based on the algorithm PIRAM and on the corresponding computer system PIRAM1.0, several Monte Carlo simulation tests have been performed. The goal is to assess in an empirical way the efficiency and sensitivity of the Minimum, Maximum and UPGMA pyramidal methods in regaining the pyramidal structure underlying in the initial data, always in terms of the expected mean value of the $\gamma$ and $\rho$ coefficients. In this study we follow the methodology introduced by F. B. Baker in [Bak74] to compare two hierarchical procedures but with several modifications other than the obvious difference between the structures considered. The main differences are the following: a) the "noises" added to the initial dissimilarities follow a normal distribution instead of being of the form $R_{b} K X$ where $R_{b}$ is the basal value, $K$ is an a prioriconstant and $X$ is a random number $-1 \leq X \leq 1$; and b) we compare the "initial" and the "final" dissimilarities but we also compare the "perturbed" dissimilarity with the "final" dissimilarity. This latter comparison makes it possible to evaluate the behaviour of the algorithm when studying arbitrary structures and to compare the spread of the distribution of the coefficients in the general case and for certain particular cases.

In the simulation tests, populations of $n=4,5,6,10,16,20$ individuals have been considered. For each of these populations, we have fixed two dissimilarities, $\delta_{\mathbf{P}}(n)$ and $\delta_{\mathrm{E}}(n)$. While the dissimilarities $\delta_{\mathbf{P}}(n)$ are chosen to be strictly Robinsonian, corresponding to strictly pyramidal structures, the dissimilarities $\delta_{\mathrm{E}}(n)$ are chosen to be enchained ultrametric. These dissimilarities are called basal dissimilarities and are displayed in Appendix A. Let $\sigma_{\mathrm{P}}(n)$ and $\sigma_{\mathrm{E}}(n)$ be the standard deviation of the corresponding dissimilarities. Next, we have perturbed 10,000 times each of the basal dissimilarities $\delta_{B}(n), B \in\{\mathbf{P}, \mathbf{E}\}$ with three random variables $\epsilon^{k}(n, i) \sim N\left(0, \sigma_{B}^{k}(n)\right)$ for $k \in\{l, m, h\}$ such that

\footnotetext{
${ }^{2}$ note that by this definition $\delta_{k}\left(h, h_{n+k}\right) \geq i\left(h_{n+k}\right)$
} 
$\sigma_{B}^{l}(n)=\sigma_{B}(n) / 3, \sigma_{B}^{m}(n)=\sigma_{B}(n)$ and $\sigma_{B}^{h}(n)=3 \sigma_{B}(n)$. In this way $\sigma_{B}^{k}(n)$ becomes a measure of the distortion degree (error level) introduced in the basal structures ( $k=l$ : (l)ow, $k=m$ (m)edium, $k=h$ (h)igh) and we obtain, for each basal dissimilarity and each $i \leq 10,000$, three new dissimilarities $\delta_{B}^{k}(n, i)=\delta_{B}(n)+\epsilon^{k}(n, i)$ with $k \in\{l, m, h\}, B \in\{\mathbf{P}, \mathbf{E}\}$, called perturbed dissimilarities.

From the perturbed dissimilarities, and by using the system PIRAM1.0, we compute the dissimilarities associated with the pyramidal structure obtained by using the method of the Maximum $\left(d_{B}^{k}(M A X, n, i)\right), \operatorname{Minimum}\left(d_{B}^{k}(M I N, n, i)\right)$ and UPGMA $\left(d_{B}^{k}(U P G M A, n, i)\right)$. Finally, the obtained Robinsonian dissimilarity is compared with both the perturbed and the basal dissimilarities. See Figure 12 for a graphical display of the whole process. We have called test $S 1$ the test in which the basal dissimilarities are strictly Robinsonian and test $S 2$ the test in which the basal dissimilarities are enchained ultrametric. The results obtained in test $S 1$ are shown in Tables 1 and 3 and the ones obtained in test $S 2$ in Tables 2 and 4.

\section{Results and conclusions}

Next we discuss the empirical results of the tests $S 1$ and $S 2$ reported in see Appendix $\mathrm{C}$ and based in 10,000 samples for each $n$.

Concerning sensitivity to data errors of the pyramidal classification methods, we should study the influence of small variations of the initial dissimilarities on the pyramidal structure obtained through the algorithm. As a first approach we can state that for each value of $n$, for each level of perturbation and each basal dissimilarity, as the distortion level increases the value of the coefficients $M_{\gamma}$ and $M_{\rho}$ decrease and the value of $S_{\gamma}$ and $S_{\rho}$ increase.

Concerning the efficiency of the methods considered, if the original structure of the population is strictly pyramidal, when looking at the comparison Basal/Final (Table 1), we can observe that the values of $\gamma$ and $\rho$ obtained for the Maximum method are higher than for any other method. In addition, the values of the standard deviation of the coefficients are also smaller for the Maximum method, independently of the number of individuals and of the perturbation level considered, as can be seen in the following table, that summarizes the behaviour of the coefficients for each error level and each method:

\begin{tabular}{llccc} 
& & \multicolumn{3}{c}{ Methods } \\
\cline { 3 - 5 } Error level & Coefficient & Maximum & UPGMA & Minimum \\
\hline low & $M_{\gamma}$ & $\approx 0.99$ & $\approx 0.75$ & $\approx 0.6$ \\
& $S_{\gamma}$ & $\approx 0.02$ & $\approx 0.1$ & $\approx 0.1$ \\
\hline medium & $M_{\gamma}$ & $0.85 \rightarrow 0.90$ & $\approx 0.75$ & $\approx 0.6$ \\
& $S_{\gamma}$ & $0.05 \rightarrow 0.17$ & $\approx 0.1$ & $\approx 0.1$ \\
\hline high & $M_{\gamma}$ & $0.37 \rightarrow 0.45$ & $0.28 \rightarrow 0.34$ & $0.16 \rightarrow 0.36$ \\
& $S_{\gamma}$ & $0.39 \rightarrow 0.13$ & $0.37 \rightarrow 0.14$ & $0.37 \rightarrow 0.10$
\end{tabular}

Concerning the average value for $M_{\rho}$ and $S_{\rho}$, at the low error level the value of $M_{\rho}$ is 0.99 and the value of $S_{\rho}$ is 0.01 for the Maximum method, while for the other two methods the values of $M_{\rho}$ are smaller and the values of $S_{\rho}$ greater (roughly 0.09). At the medium error level the values of $M_{\rho}$ for the Maximum method are $10 \%$ lower than the corresponding to the low error level and the 
values of $S_{p}$ are $10 \%$ higher; for the other two methods the values of $M_{p}$ are considerably less and the values of $M_{\rho}$ are slightly higher than for the Maximum case. At the high error level, the values of $M_{\rho}$ are less than at the low error level and the values of $S_{\rho}$ tend to be very high. So we can conclude that the Maximum method produces better results and more homogeneous. Concerning sensitivity to data errors, the Maximum method is the least sensitive; even at the medium error level it regains the initial structure yielding "good" values for the coefficients. The UPGMA method is more sensitive to data error, but at the high error level it is similar to the Maximum method. The Minimum method exhibits some difficulties in regaining the initial structure and so it is more sensitive to data errors.

Thus, from an empirical point of view we can state that if the initial structure of the population is strictly pseudo-hierarchical (not a hierarchy), the pyramidal method of the Maximum regains the pseudo-hierarchical structure underlying in the initial data in a better way than the other two methods and it is the least sensitive to the introduction of noise in the original data, so it will produce a better classification; the Minimum method is the most sensitive to data errors and the classification produced by this method will less resemble the initial structure than the classification produced by any of the other methods.

If the structure of the population is ultrametric enchained the values of $\gamma$ and $\rho$ obtained for the Minimum method are higher than the ones obtained by the other methods, as can be seen in the following table, that summarizes the results displayed in Table 2.

\begin{tabular}{llccc} 
& & \multicolumn{3}{c}{ Methods } \\
\cline { 3 - 5 } Error level & Coefficient & Maximum & UPGMA & Minimum \\
\hline low & $M_{\gamma}$ & 0.97 & 0.98 & 0.98 \\
& $S_{\gamma}$ & 0.02 & 0.02 & 0.02 \\
\hline \multirow{2}{*}{ medium } & $M_{\gamma}$ & $0.63 \rightarrow 0.91$ & $0.79 \longrightarrow 0.93$ & $0.83 \rightarrow 0.93$ \\
& $S_{\gamma}$ & $0.06 \rightarrow 0.19$ & $0.06 \rightarrow 0.19$ & $0.06 \rightarrow 0.19$ \\
\hline high & $M_{\gamma}$ & $0.21 \longrightarrow 0.48$ & $0.35 \rightarrow 0.45$ & $0.42 \rightarrow 0.49$ \\
& $S_{\gamma}$ & $0.40 \rightarrow 0.12$ & $0.56 \rightarrow 0.16$ & $0.56 \rightarrow 0.14$
\end{tabular}

We should note that although the range of values and the maximum values of $M_{\gamma}$ are similar for the three methods, the values of $M_{\gamma}$ decrease at a faster rate for the UPGMA method than did for the Minimum method, and faster for the Maximum method than for the UPGMA. In addition, the minimum value of the coefficients is smaller for the Maximum case that for the UPGMA and smaller for the UPGMA than for the Minimum. The Minimum method yields stable and homogeneous values for the low and medium error level, while at the high perturbation level the values are stable, with small deviations. So, in this case we can see that the Minimum method exhibits less sensitivity, and that a significant decrease of the values of $M_{\gamma}$ and $M_{\rho}$ and a significant increment of $S_{\gamma}$ and $S_{p}$ is only obtained when the error level is high. For instance, we can observe that for the Minimum method the value of $M_{\gamma}$ decreases a $14 \%$ when we go from the low error level to the medium error level, while in the other two cases this coefficient decreases a $17 \%$ for the UPGMA method and a $30 \%$ for the Maximum case.

Finally, we can conclude that if the structure of the initial data is enchained ultrametric, the Minimum method yields a better classification of the population. Thus in this case the most efficient method is the Minimum one. This 
method is also the least sensitive to data errors. These results follow from a more general result, proved in [CGA95], which states that the Minimum pyramidal method and the Hierarchical Minimum method are the same, and, as it is well known, the last one tends to produce enchained ultrametric structures.

Concerning the relation Perturbed/Final, the differences between the coefficients $M_{\gamma}$ and $M_{\rho}$ increase as $n$ grows, $M_{\gamma}$ being lower than $M_{\rho}$. Analyzing the values of the coefficient for each of the methods, we can see that for the Maximum method the values of $M_{\gamma}$ in the strictly pyramidal case are quite lower than the corresponding values of $M_{\rho}$

In addition, the simulation tests performed and the results obtained make it possible to draw some conclusions concerning the independence of our algorithm with respect to the initial structure of the population. Note that when the error level is high, we can consider that the perturbed dissimilarities have been generated randomly from normal distributions. In this case we can observe that the results of Tables 3 and 4 are very similar, independently of the initial structure of the data (for the Maximum method the difference between the values of $\gamma$ and $\rho$ for the cases enchained and strictly pyramidal and the highest error level is, at the most of 0.02); also, the standard deviations are quite small, so we can conclude that the algorithm is independent of the initial structure of the population. Besides, the low values of $\sigma$ suggest that the results obtained when comparing the initial and the final dissimilarities are significant, because of the concentration of the values of the coefficients around their mean value. So, we can conclude that, although some methods are better for certain initial structures, the algorithm exhibits an uniform behaviour.

At the low and medium error level, the Maximum method yields better results that the other two methods, independently of the basal structure considered. By examining the two extremal cases we can see that if the basal structure is enchained, up to medium perturbation on the data, the methods produce similar results; for the strictly pyramidal basal structure, the maximum yields very good values while the other are worse. From these facts and the previous discussion we can conclude that if we have a priori information concerning the structure of the population, indicating that the structure is enchained, then the best method we can use is the Minimum one. In absence of this kind of information or with information suggesting any other basal structure, the Maximum method will produce better results. 


\section{A Basal Dissimilarities used in tests S1 and S2}

\section{A.1 Enchained dissimilarities (Test S1)}

Figure 5: $\delta_{\mathrm{E}}(n) ; \sigma_{\mathrm{E}}(4)=0.74, \sigma_{\mathrm{E}}(5)=0.1, \sigma_{\mathrm{E}}(6)=1.25, \sigma_{\mathrm{E}}(10)=2.21$, $\sigma_{\mathrm{E}}(16)=3.63, \sigma_{\mathrm{E}}(20)=4.64$

$$
\left(\begin{array}{ccccc}
0 & 11 & \ldots & \ldots & n+9 \\
& 0 & 12 & \ldots & n+9 \\
& & \ldots & \ldots & \ldots \\
& & & 0 & n+9 \\
& & & & 0
\end{array}\right)
$$

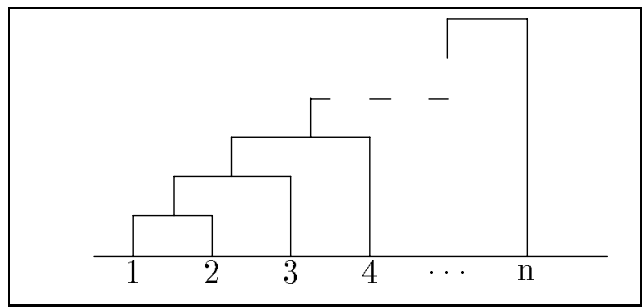

$n \in\{4,5,6,10,16,20\}$

\section{A.2 Strictly Robinsonian dissimilarities (Test S2)}

Figure $6: \delta_{\mathbf{P}}(4) ; \sigma_{\mathbf{P}}(4)=1.37$

$$
\left(\begin{array}{cccc}
0 & 11 & 14 & 14 \\
& 0 & 12 & 14 \\
& & 0 & 11 \\
& & & 0
\end{array}\right)
$$

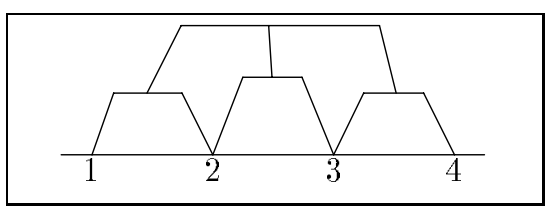

Figure $7: \delta_{\mathbf{P}}(5) ; \sigma_{\mathbf{P}}(5)=1.56$

$$
\left(\begin{array}{ccccc}
0 & 11 & 14 & 15 & 15 \\
& 0 & 12 & 15 & 15 \\
& & 0 & 11 & 14 \\
& & & 0 & 13 \\
& & & & 0
\end{array}\right)
$$

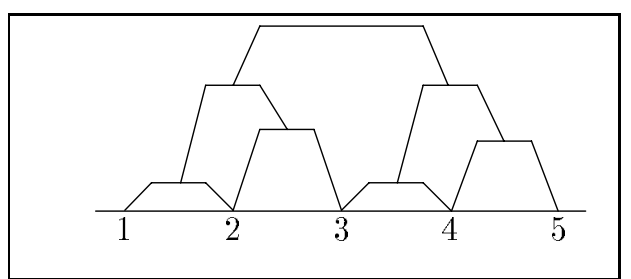

Figure $8: \delta_{\mathbf{P}}(6) ; \sigma_{\mathbf{P}}(6)=1.5$

$$
\left(\begin{array}{cccccc}
0 & 12 & 13 & 15 & 15 & 15 \\
& 0 & 11 & 15 & 15 & 15 \\
& & 0 & 11 & 14 & 14 \\
& & & 0 & 12 & 14 \\
& & & & 0 & 12 \\
& & & & & 0
\end{array}\right)
$$

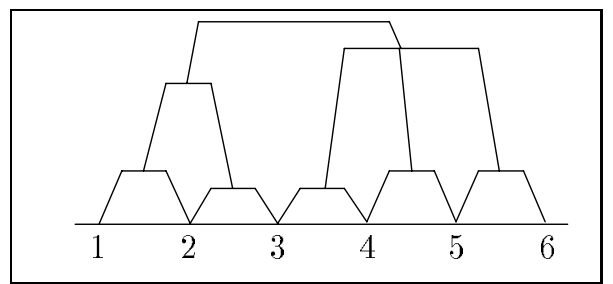


Figure $9: \delta_{\mathbf{P}}(10) ; \sigma_{\mathbf{P}}(10)=1.7$

$$
\left(\begin{array}{cccccccccc}
0 & 11 & 13 & 15 & 15 & 16 & 16 & 16 & 16 & 16 \\
& 0 & 12 & 15 & 15 & 16 & . & . & . & 16 \\
& & 0 & 11 & 13 & 16 & . & . & . & 16 \\
& & & 0 & 12 & 16 & 16 & 16 & 16 & 16 \\
& & & & 0 & 11 & 13 & 15 & 15 & 15 \\
& & & & & 0 & 12 & 15 & 15 & 15 \\
& & & & & 0 & 12 & 15 & 15 \\
& & & & & & 0 & 12 & 14 \\
& & & & & & & & 0 & 12 \\
& & & & & & & & & 0
\end{array}\right)
$$

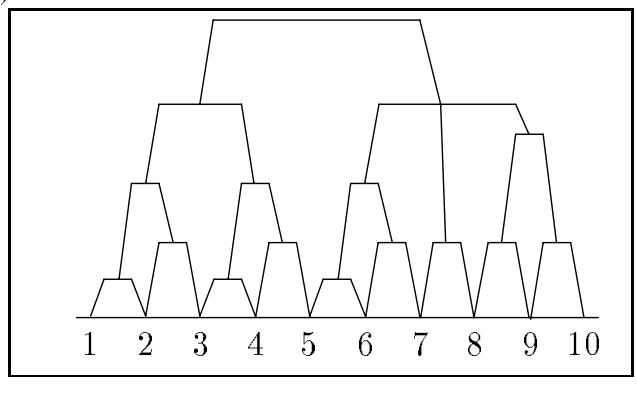

Figure $10: \delta_{\mathbf{P}}(16) ; \sigma_{\mathbf{P}}(16)=2.43$

$$
\left(\begin{array}{cccccccccccccccc}
0 & 12 & 14 & 17 & 17 & 18 & 18 & 18 & 18 & 19 & 19 & 19 & 19 & 19 & 19 & 19 \\
& 0 & 11 & 17 & 17 & 18 & . & . & 18 & 19 & . & . & . & . & . & 19 \\
& 0 & 12 & 14 & 18 & . & . & 18 & 19 & . & . & . & . & . & 19 \\
& & 0 & 11 & 18 & 18 & 18 & 18 & 19 & . & . & . & . & . & 19 \\
& & & 0 & 12 & 15 & 17 & 17 & 19 & . & . & . & . & . & 19 \\
& & & & 0 & 11 & 17 & 17 & 19 & . & . & . & . & . & 19 \\
& & & & & 0 & 12 & 13 & 19 & . & . & . & . & . & 19 \\
& & & & & & 0 & 12 & 19 & 19 & 19 & 19 & 19 & 19 & 19 \\
& & & & & & & 0 & 12 & 15 & 17 & 17 & 18 & 18 & 18 \\
& & & & & & & & 0 & 12 & 17 & 17 & 18 & 18 & 18 \\
& & & & & & & & & 0 & 11 & 15 & 17 & 17 & 17 \\
& & & & & & & & & & 0 & 12 & 17 & 17 & 17 \\
& & & & & & & & & & & 0 & 12 & 15 & 16 \\
& & & & & & & & & & & & 0 & 12 & 16 \\
& & & & & & & & & & & & & 0 & 12 \\
& & & & & & & & & & & & & & &
\end{array}\right.
$$

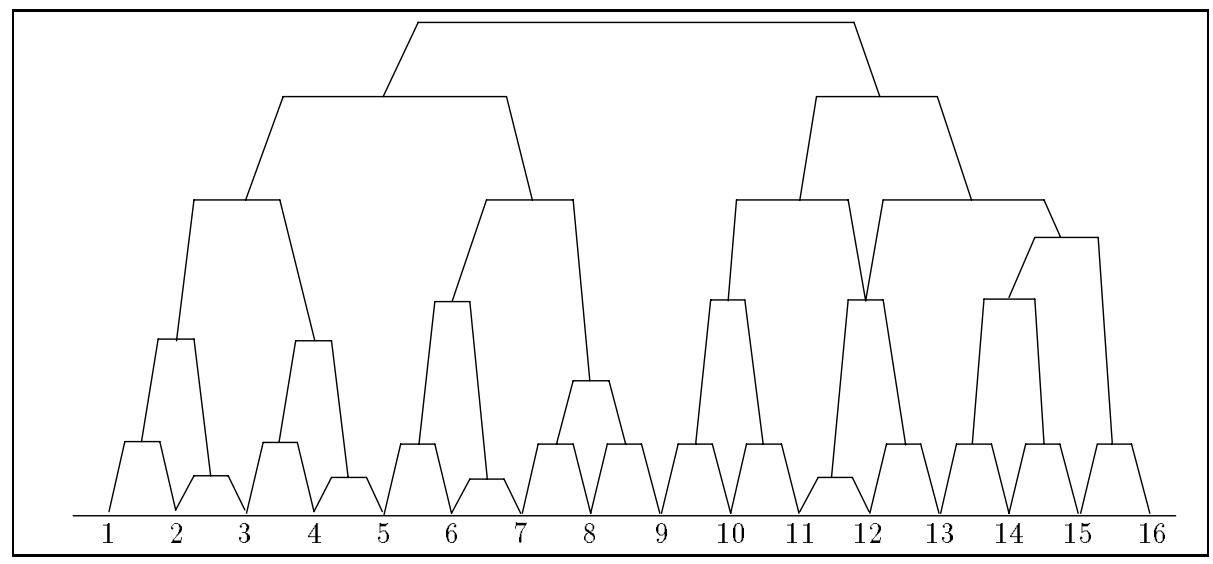


Figure 11: $\delta_{\mathbf{P}}(20) ; \sigma_{\mathbf{P}}(20)=2.4$
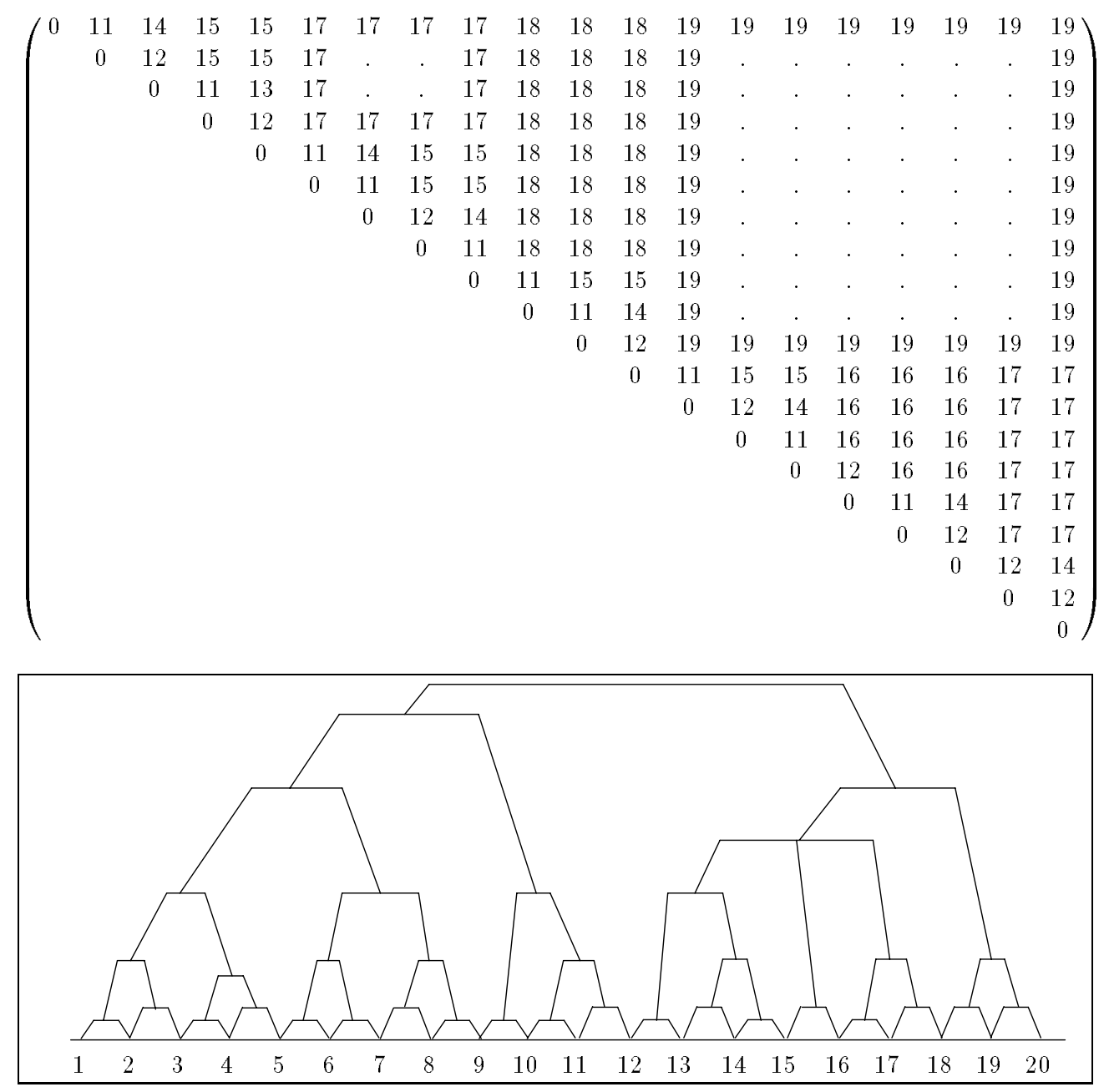


\section{B The simulation process}

Figure 12: Tests S1 and S2

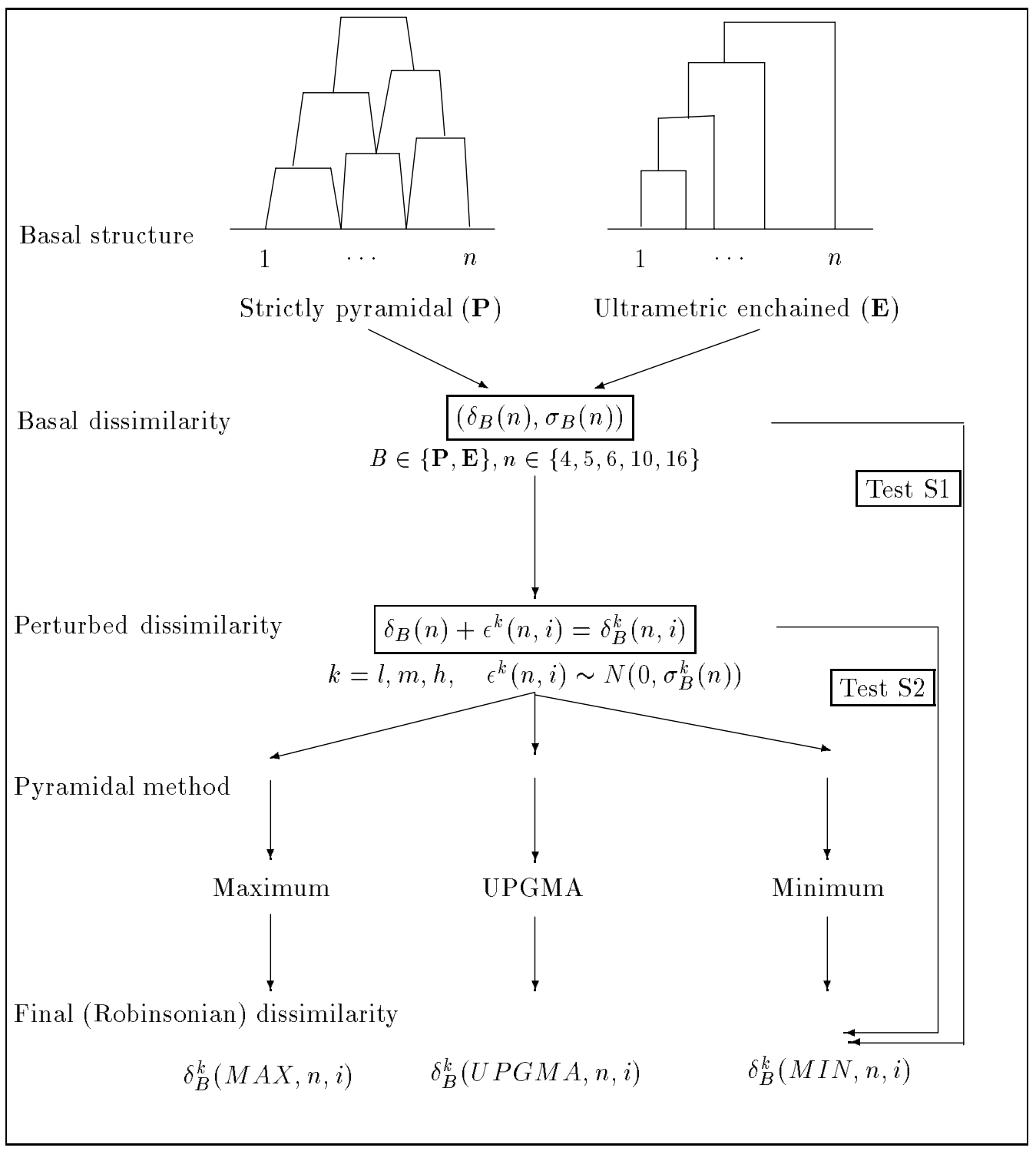




\section{Tables}

Means $\left(M_{X}\right)$ and standard deviations $\left(S_{X}\right)$ of the values of the coefficients $\gamma$ and $\rho$ calculated between the basal or perturbed dissimilarities and the final (Robinsonian)dissimilarity obtained by the methods of the Maximum, Minimum and UPGMA.

Table 1: Basal dissimilarity: Strictly Robinsonian; Comparison Basal-Final

\begin{tabular}{cc||cccc|cccc|cccc}
\hline \hline \multicolumn{1}{l|}{} & \multicolumn{1}{l||}{} & \multicolumn{4}{c}{ Maximum } & \multicolumn{4}{c}{ UPGMA } & \multicolumn{6}{c}{ Minimum } \\
\hline$n$ & $\sigma_{k}^{n}$ & $M_{\gamma}$ & $S_{\gamma}$ & $M_{\rho}$ & $S_{\rho}$ & $M_{\gamma}$ & $S_{\gamma}$ & $M_{\rho}$ & $S_{\rho}$ & $M_{\gamma}$ & $S_{\gamma}$ & $M_{\rho}$ & $S_{\rho}$ \\
\hline 4 & $\mathrm{l}$ & 1.00 & 0.03 & 0.99 & 0.01 & 0.99 & 0.07 & 0.87 & 0.06 & 0.99 & 0.07 & 0.82 & 0.07 \\
& $\mathrm{~m}$ & 0.88 & 0.17 & 0.87 & 0.13 & 0.79 & 0.28 & 0.68 & 0.22 & 0.81 & 0.28 & 0.65 & 0.20 \\
& $\mathrm{~h}$ & 0.45 & 0.42 & 0.46 & 0.39 & 0.34 & 0.45 & 0.33 & 0.37 & 0.36 & 0.47 & 0.33 & 0.37 \\
\hline 5 & $\mathrm{l}$ & 0.99 & 0.02 & 0.98 & 0.01 & 0.73 & 0.13 & 0.81 & 0.09 & 0.63 & 0.04 & 0.67 & 0.09 \\
& $\mathrm{~m}$ & 0.83 & 0.17 & 0.87 & 0.11 & 0.61 & 0.18 & 0.65 & 0.17 & 0.60 & 0.17 & 0.60 & 0.14 \\
& $\mathrm{~h}$ & 0.38 & 0.31 & 0.44 & 0.31 & 0.31 & 0.32 & 0.34 & 0.30 & 0.31 & 0.32 & 0.33 & 0.30 \\
\hline 6 & $\mathrm{l}$ & 0.99 & 0.02 & 0.98 & 0.01 & 0.49 & 0.14 & 0.52 & 0.10 & 0.45 & 0.10 & 0.47 & 0.06 \\
& $\mathrm{~m}$ & 0.85 & 0.14 & 0.88 & 0.10 & 0.53 & 0.17 & 0.56 & 0.14 & 0.48 & 0.14 & 0.49 & 0.10 \\
& $\mathrm{~h}$ & 0.37 & 0.27 & 0.43 & 0.27 & 0.28 & 0.26 & 0.31 & 0.24 & 0.27 & 0.24 & 0.29 & 0.22 \\
\hline 10 & $\mathrm{l}$ & 0.99 & 0.01 & 0.98 & 0.01 & 0.76 & 0.10 & 0.77 & 0.07 & 0.59 & 0.16 & 0.64 & 0.08 \\
& $\mathrm{~m}$ & 0.87 & 0.08 & 0.66 & 0.15 & 0.68 & 0.10 & 0.52 & 0.17 & 0.56 & 0.10 & 0.57 & 0.11 \\
& $\mathrm{~h}$ & 0.40 & 0.19 & 0.29 & 0.21 & 0.33 & 0.18 & 0.22 & 0.18 & 0.27 & 0.16 & 0.25 & 0.18 \\
\hline 16 & $\mathrm{l}$ & 0.98 & 0.01 & 0.98 & 0.08 & 0.77 & 0.05 & 0.59 & 0.16 & 0.62 & 0.08 & 0.63 & 0.06 \\
& $\mathrm{~m}$ & 0.90 & 0.05 & 0.73 & 0.11 & 0.74 & 0.06 & 0.56 & 0.16 & 0.60 & 0.09 & 0.57 & 0.08 \\
& $\mathrm{~h}$ & 0.41 & 0.14 & 0.29 & 0.18 & 0.37 & 0.14 & 0.18 & 0.13 & 0.27 & 0.12 & 0.25 & 0.10 \\
\hline 20 & $\mathrm{l}$ & 0.98 & 0.01 & 0.99 & 0.00 & 0.78 & 0.07 & 0.80 & 0.04 & 0.53 & 0.15 & 0.61 & 0.09 \\
& $\mathrm{~m}$ & 0.85 & 0.10 & 0.91 & 0.05 & 0.72 & 0.10 & 0.76 & 0.06 & 0.54 & 0.17 & 0.60 & 0.09 \\
& $\mathrm{~h}$ & 0.26 & 0.15 & 0.38 & 0.13 & 0.28 & 0.18 & 0.36 & 0.14 & 0.16 & 0.11 & 0.25 & 0.10
\end{tabular}

Table 2: Basal dissimilarity: Enchained; Comparison Basal-Final

\begin{tabular}{cc||cccc|cccc|cccc}
\hline \hline \multicolumn{1}{c||}{} & \multicolumn{4}{c||}{ Maximum } & \multicolumn{4}{c}{ UPGMA } & \multicolumn{4}{c}{ Minimum } \\
\hline$n$ & $\sigma_{k}^{n}$ & $M_{\gamma}$ & $S_{\gamma}$ & $M_{p}$ & $S_{p}$ & $M_{\gamma}$ & $S_{\gamma}$ & $M_{p}$ & $S_{p}$ & $M_{\gamma}$ & $S_{\gamma}$ & $M_{p}$ & $S_{p}$ \\
\hline 4 & $\mathrm{l}$ & 0.95 & 0.07 & 0.99 & 0.01 & 0.84 & 0.13 & 0.88 & 0.06 & 0.99 & 0.05 & 0.83 & 0.07 \\
& $\mathrm{~m}$ & 0.95 & 0.07 & 0.97 & 0.05 & 0.79 & 0.17 & 0.78 & 0.17 & 0.88 & 0.19 & 0.76 & 0.16 \\
& $\mathrm{~h}$ & 0.95 & 0.07 & 0.95 & 0.07 & 0.76 & 0.20 & 0.73 & 0.20 & 0.79 & 0.21 & 0.73 & 0.19 \\
\hline 5 & $\mathrm{l}$ & 0.96 & 0.04 & 0.99 & 0.01 & 0.66 & 0.16 & 0.82 & 0.09 & 0.55 & 0.10 & 0.67 & 0.10 \\
& $\mathrm{~m}$ & 0.89 & 0.09 & 0.95 & 0.05 & 0.62 & 0.18 & 0.71 & 0.16 & 0.61 & 0.19 & 0.68 & 0.16 \\
& $\mathrm{~h}$ & 0.84 & 0.10 & 0.89 & 0.08 & 0.64 & 0.18 & 0.68 & 0.18 & 0.65 & 0.19 & 0.67 & 0.17 \\
\hline 6 & $\mathrm{l}$ & 0.94 & 0.04 & 0.99 & 0.01 & 0.44 & 0.14 & 0.54 & 0.11 & 0.42 & 0.11 & 0.5 & 0.07 \\
& $\mathrm{~m}$ & 0.85 & 0.08 & 0.93 & 0.05 & 0.53 & 0.17 & 0.63 & 0.15 & 0.51 & 0.17 & 0.59 & 0.13 \\
& $\mathrm{~h}$ & 0.75 & 0.10 & 0.84 & 0.08 & 0.56 & 0.15 & 0.64 & 0.15 & 0.54 & 0.16 & 0.61 & 0.14 \\
\hline 10 & $\mathrm{l}$ & 0.84 & 0.04 & 0.98 & 0.01 & 0.62 & 0.10 & 0.76 & 0.07 & 0.49 & 0.16 & 0.65 & 0.08 \\
& $\mathrm{~m}$ & 0.67 & 0.08 & 0.86 & 0.05 & 0.50 & 0.11 & 0.67 & 0.09 & 0.43 & 0.13 & 0.60 & 0.10 \\
& $\mathrm{~h}$ & 0.50 & 0.08 & 0.70 & 0.07 & 0.40 & 0.10 & 0.55 & 0.11 & 0.33 & 0.12 & 0.50 & 0.12 \\
\hline 16 & $\mathrm{l}$ & 0.78 & 0.04 & 0.98 & 0.01 & 0.61 & 0.08 & 0.76 & 0.05 & 0.48 & 0.15 & 0.64 & 0.08 \\
& $\mathrm{~m}$ & 0.57 & 0.07 & 0.84 & 0.03 & 0.47 & 0.07 & 0.68 & 0.06 & 0.38 & 0.11 & 0.59 & 0.08 \\
& $\mathrm{~h}$ & 0.34 & 0.06 & 0.59 & 0.05 & 0.30 & 0.06 & 0.48 & 0.07 & 0.22 & 0.07 & 0.41 & 0.09 \\
\hline 20 & $\mathrm{l}$ & 0.80 & 0.04 & 0.98 & 0.00 & 0.63 & 0.07 & 0.78 & 0.04 & 0.44 & 0.15 & 0.62 & 0.08 \\
& $\mathrm{~m}$ & 0.58 & 0.06 & 0.83 & 0.03 & 0.49 & 0.07 & 0.68 & 0.05 & 0.38 & 0.12 & 0.58 & 0.08 \\
& $\mathrm{~h}$ & 0.28 & 0.05 & 0.54 & 0.05 & 0.26 & 0.06 & 0.45 & 0.06 & 0.18 & 0.06 & 0.37 & 0.08
\end{tabular}


Table 3: Basal dissimilarity: Strictly Robinsonian; Comparison PerturbedFinal

\begin{tabular}{cc||cccc|cccc|cccc}
\hline \hline \multicolumn{1}{l|}{} & & \multicolumn{4}{c||}{ Maximum } & \multicolumn{4}{c}{ UPGMA } & \multicolumn{4}{c}{ Minimum } \\
\hline$n$ & $\sigma_{k}^{n}$ & $M_{\gamma}$ & $S_{\gamma}$ & $M_{\rho}$ & $S_{\rho}$ & $M_{\gamma}$ & $S_{\gamma}$ & $M_{\rho}$ & $S_{\rho}$ & $M_{\gamma}$ & $S_{\gamma}$ & $M_{\rho}$ & $S_{\rho}$ \\
\hline 4 & $\mathrm{l}$ & 1.00 & 0.00 & 0.98 & 0.01 & 1.00 & 0.00 & 0.99 & 0.01 & 1.00 & 0.00 & 0.99 & 0.01 \\
& $\mathrm{~m}$ & 0.91 & 0.15 & 0.89 & 0.09 & 0.93 & 0.19 & 0.93 & 0.10 & 0.93 & 0.19 & 0.93 & 0.10 \\
& $\mathrm{~h}$ & 0.48 & 0.40 & 0.49 & 0.37 & 0.42 & 0.56 & 0.47 & 0.50 & 0.42 & 0.56 & 0.47 & 0.49 \\
\hline 5 & $\mathrm{l}$ & 1.00 & 0.00 & 0.98 & 0.01 & 1.00 & 0.00 & 0.99 & 0.01 & 1.00 & 0.00 & 0.99 & 0.01 \\
& $\mathrm{~m}$ & 0.85 & 0.14 & 0.88 & 0.08 & 0.90 & 0.17 & 0.92 & 0.08 & 0.91 & 0.17 & 0.92 & 0.08 \\
& $\mathrm{~h}$ & 0.42 & 0.30 & 0.47 & 0.29 & 0.43 & 0.42 & 0.47 & 0.39 & 0.44 & 0.43 & 0.48 & 0.38 \\
\hline 6 & $\mathrm{l}$ & 1.00 & 0.01 & 0.98 & 0.01 & 1.00 & 0.00 & 0.99 & 0.01 & 1.00 & 0.00 & 0.99 & 0.01 \\
& $\mathrm{~m}$ & 0.82 & 0.12 & 0.87 & 0.07 & 0.89 & 0.15 & 0.91 & 0.08 & 0.89 & 0.16 & 0.92 & 0.07 \\
& $\mathrm{~h}$ & 0.38 & 0.25 & 0.44 & 0.26 & 0.42 & 0.35 & 0.47 & 0.34 & 0.44 & 0.37 & 0.48 & 0.34 \\
\hline 10 & $\mathrm{l}$ & 0.96 & 0.02 & 0.98 & 0.01 & 0.99 & 0.03 & 0.99 & 0.01 & 0.99 & 0.02 & 0.99 & 0.00 \\
& $\mathrm{~m}$ & 0.73 & 0.10 & 0.84 & 0.07 & 0.84 & 0.11 & 0.91 & 0.05 & 0.86 & 0.10 & 0.92 & 0.04 \\
& $\mathrm{~h}$ & 0.30 & 0.17 & 0.38 & 0.18 & 0.39 & 0.24 & 0.46 & 0.23 & 0.45 & 0.24 & 0.53 & 0.23 \\
\hline 16 & $\mathrm{l}$ & 0.94 & 0.02 & 0.98 & 0.01 & 0.97 & 0.03 & 0.99 & 0.00 & 0.98 & 0.03 & 0.99 & 0.00 \\
& $\mathrm{~m}$ & 0.66 & 0.11 & 0.79 & 0.09 & 0.80 & 0.08 & 0.90 & 0.04 & 0.83 & 0.08 & 0.92 & 0.03 \\
& $\mathrm{~h}$ & 0.23 & 0.14 & 0.31 & 0.14 & 0.35 & 0.18 & 0.44 & 0.18 & 0.46 & 0.17 & 0.56 & 0.17 \\
\hline 20 & $\mathrm{l}$ & 0.93 & 0.02 & 0.98 & 0.01 & 0.96 & 0.03 & 0.99 & 0.00 & 0.97 & 0.02 & 0.99 & 0.00 \\
& $\mathrm{~m}$ & 0.63 & 0.11 & 0.77 & 0.09 & 0.79 & 0.07 & 0.90 & 0.04 & 0.83 & 0.06 & 0.93 & 0.02 \\
& $\mathrm{~h}$ & 0.21 & 0.12 & 0.29 & 0.12 & 0.35 & 0.16 & 0.43 & 0.16 & 0.49 & 0.14 & 0.60 & 0.14
\end{tabular}

Table 4: Basal dissimilarity: Enchained; Comparison Perturbed-Final

\begin{tabular}{cc||cccc|cccc|cccc}
\hline \hline \multicolumn{1}{c||}{} & \multicolumn{4}{c||}{ Maximum } & \multicolumn{4}{c}{ UPGMA } \\
\hline$n$ & $\sigma_{k}^{n}$ & $M_{\gamma}$ & $S_{\gamma}$ & $M_{\rho}$ & $S_{\rho}$ & $M_{\gamma}$ & $S_{\gamma}$ & $M_{\rho}$ & $S_{\rho}$ & $M_{\gamma}$ & $S_{\gamma}$ & $M_{\rho}$ & $S_{\rho}$ \\
\hline 4 & $\mathrm{l}$ & 1.00 & 0.00 & 1.00 & 0.00 & 1.00 & 0.00 & 0.99 & 0.01 & 1.00 & 0.00 & 0.99 & 0.01 \\
& $\mathrm{~m}$ & 0.99 & 0.04 & 0.98 & 0.03 & 0.93 & 0.14 & 0.90 & 0.09 & 0.93 & 0.13 & 0.90 & 0.90 \\
& $\mathrm{~h}$ & 0.96 & 0.07 & 0.96 & 0.06 & 0.78 & 0.21 & 0.75 & 0.19 & 0.79 & 0.21 & 0.75 & 0.18 \\
\hline 5 & $\mathrm{l}$ & 0.98 & 0.02 & 0.99 & 0.01 & 1.00 & 0.01 & 0.98 & 0.01 & 1.00 & 0.00 & 0.98 & 0.01 \\
& $\mathrm{~m}$ & 0.94 & 0.07 & 0.97 & 0.03 & 0.86 & 0.13 & 0.88 & 0.08 & 0.86 & 0.13 & 0.88 & 0.07 \\
& $\mathrm{~h}$ & 0.86 & 0.09 & 0.91 & 0.08 & 0.65 & 0.18 & 0.70 & 0.16 & 0.66 & 0.19 & 0.69 & 0.16 \\
\hline 6 & $\mathrm{l}$ & 0.97 & 0.02 & 0.99 & 0.01 & 1.00 & 0.01 & 0.98 & 0.01 & 1.00 & 0.01 & 0.98 & 0.01 \\
& $\mathrm{~m}$ & 0.88 & 0.07 & 0.94 & 0.04 & 0.81 & 0.11 & 0.87 & 0.07 & 0.81 & 0.11 & 0.86 & 0.07 \\
& $\mathrm{~h}$ & 0.77 & 0.10 & 0.86 & 0.08 & 0.58 & 0.15 & 0.67 & 0.14 & 0.57 & 0.16 & 0.65 & 0.15 \\
\hline 10 & $\mathrm{l}$ & 0.93 & 0.02 & 0.98 & 0.01 & 0.95 & 0.03 & 0.97 & 0.01 & 0.96 & 0.02 & 0.98 & 0.01 \\
& $\mathrm{~m}$ & 0.74 & 0.07 & 0.88 & 0.04 & 0.72 & 0.07 & 0.84 & 0.05 & 0.71 & 0.07 & 0.84 & 0.04 \\
& $\mathrm{~h}$ & 0.53 & 0.09 & 0.71 & 0.08 & 0.44 & 0.10 & 0.58 & 0.10 & 0.41 & 0.11 & 0.56 & 0.10 \\
\hline 16 & $\mathrm{l}$ & 0.89 & 0.02 & 0.97 & 0.01 & 0.91 & 0.02 & 0.97 & 0.00 & 0.91 & 0.02 & 0.97 & 0.00 \\
& $\mathrm{~m}$ & 0.63 & 0.08 & 0.81 & 0.06 & 0.66 & 0.05 & 0.82 & 0.04 & 0.66 & 0.04 & 0.82 & 0.03 \\
& $\mathrm{~h}$ & 0.36 & 0.07 & 0.58 & 0.06 & 0.35 & 0.07 & 0.52 & 0.07 & 0.32 & 0.07 & 0.48 & 0.08 \\
\hline 20 & $\mathrm{l}$ & 0.88 & 0.02 & 0.97 & 0.01 & 0.90 & 0.02 & 0.97 & 0.00 & 0.90 & 0.01 & 0.97 & 0.00 \\
& $\mathrm{~m}$ & 0.59 & 0.08 & 0.78 & 0.06 & 0.65 & 0.04 & 0.81 & 0.03 & 0.64 & 0.03 & 0.82 & 0.02 \\
& $\mathrm{~h}$ & 0.31 & 0.06 & 0.53 & 0.05 & 0.32 & 0.07 & 0.50 & 0.06 & 0.30 & 0.06 & 0.45 & 0.07
\end{tabular}




\section{References}

[Bak74] F.B. Baker. Stability of Two Hierarchical Grouping Techniques. Case I: Sensitivity to Data Errors. Journal of the American Statistical Association, 69(346):440-445, 1974.

[BD85] P. Bertrand and E. Diday. A Visual Representation of the Compatibility between an Order and a Dissimilarity Index: the Pyramids. Computational Statistics Quarterly, 2(Issue 1):31-41, 1985.

[Ber86] P. Bertrand. Etude de la Représentation Pyramidale. Thèse de 3ème. cycle, Universitè de Paris-Dauphine, 1986.

[BH75] F.B. Baker and L.J. Hubert. Measuring the Power of Hierarchical Cluster Analysis. Journal of the American Statistical Association, $70: 31-38,1975$.

[Boc84] H.H. Bock. Statistical Testing and Evaluation Methods in Cluster Analysis. In Proceedings of the Indian Statistical Institute Golden Jubilee, International Conference on Statistics: Applications and New Directions, 1984.

[CA92] C. Capdevila and A. Arcas. Sobre un Algoritmo de Clasificación Mediante Grafos Piramidales. In Proceedings of the XX Reunion Nacional de Estadística e Investigación Operativa, Cáceres, 1992. Universidad de Extremadura.

[CA94] C. Capdevila and A. Arcas. Modelos de Representación Piramidal. Algunas Técnicas de la Bondad de Ajuste. In XXI Congreso Nacional de Estadística e Investigación operativa, pages 87-88, Calella, Spain, 1994.

[CA95a] C. Capdevila and A. Arcas. Empirical Signifiance Test of the Goodness-of-Fit for some Pyramidal Clustering Procedures. Questiió, 19:123-130, 1995.

[CA95b] C. Capdevila and A. Arcas. Piramides Indexadas y Disimilaridades Piramidales. Questioó, 19:131-151, 1995.

[Cap93] C. Capdevila. Contribuciones al Estudio del Problema de la Clasificación Mediante Grafos Piramidales. Tesis de doctorado en matemáticas, Universitat de Barcelona, 1993.

[CGA95] C. Capdevila, A.J. Gil, and A. Arcas. Algunas Propiedades de un Algoritmo de Clasificación Piramidal. Estadística Española, 37:101126, 1995 .

[CGA96] C. Capdevila, A.J. Gil, and A. Arcas. Efficiency and Sensitivity of three Pyramidal Classification Methods. In A. Prat and E. Ripoll, editors, Proceedings in Computational Statistics COMPSTAT'96, Barcelona, 1996. Universitat Politècnica de Catalunya. 
[DF88] C. Durand and B. Fichet. One-to-one Correspondences in Pyramidal Representation: A Unified Approach. In H.H. Bock, editor, Classification and Related Methods of Data Analysis. North-Holland, Amsterdam, 1988.

[Did86a] E. Diday. Orders and Overlapping Clusters by Pyramids. In J. de Leeuw, W. Heiser, J. Meulman, and F. Critchley, editors, Multidimensional Data Analysis, pages 201-234. Leiden: DSWO, 1986.

[Did86b] E. Diday. Une Représentation Visuelle des Classes Empietantes: Les Pyramides. Rairo: Analyse des Données, 52:475-526, 1986.

[Dur86] C. Durand. Sur la Représentation Pyramidale en Analyse des Données. Mémoire de DEA en Mathématiques Appliquées, Université de Provence, Marseille, 1986.

[Dur88] C. Durand. Une Approximation de Robinson Inférieur Maximale. Rapport de Recherche 88-02, Laboratoire de Mathématiques Appliquées et Informatique, Université de Provence, Marseille, 1988.

[GC96] A.J. Gil and C. Capdevila. A New Program of Pyramidal Classification. In A. Prat and E. Ripoll, editors, Proceedings in Computational Statistics COMPSTAT'96, Barcelona, 1996. Universitat Politècnica de Catalunya.

[GCA94a] A.J. Gil, C. Capdevila, and A. Arcas. A program of Pyramidal Classification. In XXI Congreso Nacional de Estadística e Investigación operativa, pages 350-351, Calella, Spain, 1994.

[GCA94b] A.J. Gil, C. Capdevila, and A. Arcas. Automatización de los métodos piramidales de clasificación y representación de datos. WorkingPaper 94/3, Diplomatura en Ciències Empresarials, Universitat Pompeu Fabra. Barcelona, 1994.

[GK54] L.A. Goodman and W.H. Kruskal. Measures of Association for Cross-Classification. Journal of the American Statistical Association, 49:732-764, 1954.

[Gor96] A.D. Gordon. Cluster validation. In IFCS-96, Kobe, 1996.

[GS94] W. Gaul and M. Schader. Pyramidal Classification based on Incomplete Dissimilarity Data. Journal of Classification, 11:171-193, 1994.

[Hub74] L.J. Hubert. Approximate Evaluation Techniques for the Single-link and Complete-link Hierarchical Procedures. Journal of the American Statistical Association, 69:698-704, 1974.

[JS71] N. Jardine and R. Sibson. Mathematical Taxonomy. Wiley, New York, 1971.

[KR90] L Kaufman and P.J. Rousseeuw. Finding groups in data. An introduction to cluster analysis. Wiley, New York, 1990. 
[Man94] Bryan F.J. Manly. Multivariate statistical methods. A primer. Chapman and Hall, London, 1994.

[SM58] R.R Sokal and C.D. Michener. A statistical method for evaluating systematic relatioship. SCI. BULL. 38, Kansas University, 1958.

[SR62] R.R. Sokal and J.F. Rohlf. The Comparison of Dendrograms by Objective Methods. Taxon, 11:33-39, 1962. 